\title{
Journal of Clinical and Nursing Research
}

\author{
Research Article
}

\section{The Influence of Neuman Theory Based Stress Management on the Level of Benefit Finding in Postoperative Breast Cancer Patients}

\author{
Qin Dai ${ }^{1,2}$, Huili Hang ${ }^{2 *}$, Chunxiu $\mathrm{He}^{2}$, Yuwen Zhou ${ }^{2}$, Jing Zhang ${ }^{2}$ \\ ${ }^{1}$ Medical School of Nantong University, Nantong 226001, Jiangsu Province, China; \\ ${ }^{2}$ Taizhou Second People's Hospital, Taizhou 225500, Jiangsu Province, China
}

Funding: Jiangyan District Science and Technology Special Support Fund Project (SSF201830011).

\begin{abstract}
Objective: To explore the influence of stress management program based on Neuman theory on the level of benefit finding in postoperative breast cancer patients. Methods: Using convenient sampling method, 60 postoperative breast cancer patients in the tumor ward of a hospital were selected and randomly assigned into observation group $(n=30)$ and control group $(n=30)$. The control group adopts the conventional postoperative psychological nursing program, and the observation group adopts the stress management program based on Neuman theory for psychological nursing. The differences in benefit finding, life quality, and medical coping strategies between the two groups before and after nursing were compared. Results: After the implementation of the two nursing programs, the level of benefit finding, life quality, and medical coping strategies of patients in the observation group were higher than those in the control group $\left(t_{1}=3.996, t_{2}=4.662, t_{3}=4.113, P<0.05\right)$; there was a statistically significant difference in the level of benefit finding, life quality, and medical coping strategies of patients before and after the implementation of the two nursing programs $(P<0.05)$. Conclusions: The stress management strategy based on Neuman theory can improve patients' state of psychological stress coping, play a positive role in guiding the psychology of patients after breast cancer surgery, and can be used as a reference for the psychological nursing program of postoperative patients.
\end{abstract}

Keywords: Neuman theory; Breast cancer postoperation;
Benefit finding

Publication date: November, 2020

Publication online: 30 November, 2020

"Corresponding author: Chunxiu He, 13952606766 @163.com

Breast cancer is one of the most common malignant tumors in women. Currently, patients are often treated with modified radical mastectomy. After surgery, patients are prone to psychological symptoms such as anxiety and depression, which seriously affect the prognosis of patients ${ }^{[2]}$. Benefit finding not only directly affect the patient's life quality but also improve the patients' depression, thereby influencing their life quality indirectly. Related research on the Neuman system model ${ }^{[3]}$ regard people as a whole, multi-dimensional open system, constantly interacting with stress (stressors) in the external environment, and maintain the balance and integrity of the entire system through its own defense system. When someone's defense system or response ability cannot maintain the balance of the entire system, a series of pathological reactions and even crises of physiological and psychological nature will occur. The stressor can be intervened at this time. This theory has gradually been applied to many nursing work and has obtained good results.

\section{Subjects and Methods}

\subsection{Study Subjects}

Using convenient sampling method, 60 postoperative 
breast cancer patients from the tumor ward of a tertiary general hospital in Taizhou City, Jiangsu Province were selected as the research subjects. Inclusion criteria: women who were diagnosed with breast cancer for the first time; breast cancer did not metastasize; patients voluntarily participated in the study and had given informed consent. Exclusion criteria: Patients with mental illness; Patients with other organ or system diseases. Sixty subjects were randomly divided into observation group and control group. Each group had 30 postoperative breast cancer patients. Before the intervention, there was no significant difference in the level of benefit finding, life quality, and medical coping strategies between the two groups. Significance $(P>0.05)$.

\subsection{Methods}

\subsubsection{Control Group}

Conventional psychological nursing interventions was administered to the 30 cases of postoperative breast cancer patients in the control group, including disease knowledge education, postoperative points of attention, points of attention related to home diseases, timely observation of changes in the patients' mental state, relevant psychological care measures and etc. The program lasted a month.

\subsubsection{Observation Group}

On the basis of the conventional psychological nursing intervention of the control group, the stress management strategy was developed based on the Neuman theory for the 30 breast cancer patients in the observation group, and a total of 1 month long intervention was conducted. During the hospitalization period, rounds were conducted every Monday night. During the discharge period, a WeChat group was created to provide online guidance and intervention every Monday. The specific intervention measures are as follow (vide infra).

Assessing the Stressors of the 30 Postoperative Breast Cancer Patients in the Observation Group with Neuman Theory. Based on Neuman theory, the stressors and destabilizing factors of the observation group after breast cancer surgery were found: the patient's own factors, disease treatment factors, and family issues factors.

Developing Stress Management Strategies for Postoperative Breast Cancer Patients: (1)Multichannel and all-round health education enables patients to better understand the breast cancer disease itself and enhance patients' confidence in the prognosis. With easy-to-understand language, a picture album on breast cancer related knowledge and postoperative rehabilitation exercises, and microvideos on introduction of breast cancer surgery methods and postoperative precautions, etc were produced.

A small lecture on breast cancer knowledge and health was held once a week and patients were raised with frequently asked questions to achieve a unified popularization of related questions and focus points. At the end of the small lecture, patients were encouraged to communicate with each other and encourage each other, so that they can gain a certain sense of identity through empathy, reducing their loneliness and enhance their confidence in conquering the disease.

The patients were divided into 3 groups according to their surgical method, place of residence, age group, and education level, etc and a WeChat group was created for each group. Each group will be introduced the corresponding breast cancer surgery methods and postoperative precautions with microvideo playback and how to manage self-image after breast cancer surgery to help patients gain knowledge of their own surgical methods, postoperative precautions, and rehabilitation exercises.

(2)Strengthening of the extended care of patients after breast cancer surgery, timely assessment of the mental state of patients, enhancing family support of patients, and correcting patients' self-image disorder help patients confront the disease firmly and promote harmony in the patients' families.

During hospitalization, the patient's family members, especially the patient's spouse, were encouraged to actively participate in the health lectures and join the WeChat group etc. This allows the patient's family members to understand the wife's ongoing worries about the disease, give the wife certain encouragement with suitable points, especially as most of the patients are worried about the postoperative image change. The participation of the patient's family can give some positive encouragement to the patient.

After discharge, home visits for extended nursing care were conducted according to the patient's wishes and needs. The patient's intubation and rehabilitation exercises were attended to, and assessments of the 
patient's psychological dynamics were conducted to provide timely answers for the patients. Patients were encouraged to communicate with relatives and friends and listen more to smoothing music. Where stamina allows, patients were advised to perform mountain climbing, dancing, and etc. appropriately, to strengthen the patient's social support and help avoid bad emotions.

\subsection{Observation Indicators}

\subsubsection{Benefit Finding Scale}

Benefit Finding Scale - Chinese (BFS-C)consists of a total of 5 terms, 17 items, with a 5-level scoring scale from 1 (nothing at all) to 5 (very many). The higher the score, the more benefits the patient perceives on the item ${ }^{[4]}$.

\subsubsection{Quality of Life Instrument for Cancer Patients-Breast Cancer (QLICP-BR)}

The scale includes 6 physical function dimensions (PH), 12 psychological function dimensions (PS), 8 symptoms and side effects dimensions (ST), 10 social function dimensions (SO), and 1 overall health status, totalling 37 items. Each scoring is based on no (1), a little (2), some (3), equivalent (4), very (5) points.
When scoring a reverse entry, the score is reversed in the positive scoring order. Higher score indicates that the patient has better effects on this item ${ }^{[5]}$.

\subsubsection{Medical Coping Modes Questionnaire(MCMQ)}

The Chinese version of the MCMQ has a total of 20 items, and each item is scored at levels from 1 to 4 , with total score ranging from 20 to 80 points. There are 8 items that require reverse scoring. It is divided into three dimensions: "confront", "avoid" and "surrender". The "confront" dimension is scored in item $1,2,5,10,12,15,16$, and 19 with cumulative scores, and the "avoid" dimension is scored in item $3,7,8,9,11,14$, and 17 with cumulative scores, and the "surrender" dimension is scored in item 4, 6, 13, 18 , and 20 with cumulative scores as well. The higher the score of a dimension, the stronger its coping strategies are ${ }^{[6]}$.

\section{Results}

Before and after the intervention, the total scores of benefit finding, life quality, and medical coping strategies between the control group and the observation group were compared. The differences were statistically significant $(P<0.05)$, See Table 1 .

Table 1. Comparison of Benefit Finding, Life Quality and Medical Stress Coping Strategies Scores between the Control Group and the Observation Group Before and After Intervention $(\bar{x} \pm s$, Scores $)$

\begin{tabular}{|c|c|c|c|c|c|c|}
\hline Term & Group & Sample size & Before Intervention & After Intervention & $t$ & $P$ \\
\hline \multirow{2}{*}{$\begin{array}{l}\text { Benefit Finding } \\
\text { Scores }\end{array}$} & Control & 30 & $37.16 \pm 5.31$ & $40.22 \pm 6.14$ & -3.071 & $0.001^{*}$ \\
\hline & Observation & 30 & $37.54 \pm 5.42$ & $44.17 \pm 6.33$ & -6.224 & $0.001^{*}$ \\
\hline$t$ & & & -0.491 & -3.996 & & \\
\hline$P$ & & & 0.792 & $0.001^{*}$ & & \\
\hline \multirow{2}{*}{ Life Quality Scores } & Control & 30 & $67.41 \pm 7.24$ & $73.25 \pm 8.41$ & -5.844 & $0.001^{*}$ \\
\hline & Observation & 30 & $66.98 \pm 7.03$ & $77.91 \pm 7.26$ & -10.032 & $0.000^{*}$ \\
\hline$t$ & & & 0.431 & -4.662 & & \\
\hline$P$ & & & 0.647 & $0.001 *$ & & \\
\hline Stress Coping & Control & 30 & $38.97 \pm 3.17$ & $42.29 \pm 3.56$ & -3.334 & $0.001^{*}$ \\
\hline Scores & Observation & 30 & $38.06 \pm 3.06$ & $46.18 \pm 4.01$ & -8.024 & $0.000^{*}$ \\
\hline$t$ & & & 0.912 & -4.113 & & \\
\hline$P$ & & & 0.944 & $0.000 *$ & & \\
\hline
\end{tabular}

\section{Discussions}

Due to the process of treatment and diagnosis of breast cancer, and changes in female characteristics after surgery, patients are more likely to develop depression, anxiety and other emotions, which affect surgery and recovery. These poor mental states can easily affect the patient's coping strategies of the disease and life quality. Related research ${ }^{[7]}$ shows that positive mentality and emotions of breast cancer patients can improve the life quality of patients after surgery. Benefit finding, as an important research topic of positive mentality, is conducive to patients to discover the positive significance of the diseases and promote physical and psychological recovery. However, there is currently no interventional study on benefit finding for breast cancer patients in China.

Therefore, in this study, a series of stress management strategies were formulated based on 
Neuman theory, focusing on discovering the negative mentality of breast cancer patients, and gradually improving the psychological state of patients, so that patients can actively face the disease and achieve a balance between internal and external environments. The results of this study show that after intervention with the stress management strategy based on the aforementioned Neuman theory was given to the patients in the observation group, the benefit finding level, medical coping level, and life quality of the observation group were significantly higher than those of the control group, indicating that this stress management strategy played a certain positive role. Huarong Lin's related research ${ }^{[8]}$ also shows that the Neuman care model enables patients to confront the disease correctly, reduce the fear of the disease, and enhance treatment confidence, thereby reducing anxiety, depression and other bad emotions and maintaining a good mental state.

In conclusion, the stress management strategy based on Neuman theory can make patients feel positive, affect the patient's benefit finding level, medical coping strategies and life quality, hence having certain clinical significance. Nevertheless, this study has certain shortcomings. It is hoped that a systematic and comprehensive strategy for stress management of postoperative breast cancer patients can be established in the future to improve the life quality of the patients.

\section{References}

[1] Qiao LZ. The effect of humanistic care on post-operative psychological stress and self-care ability of patients with modified radical mastectomy[J]. Henan Medical Research,2020,29(15):2868-2869.

[2] Yu PW. Development trajectory and function of benefit discovery for female breast cancer patients [D]. Central South University, 2014.

[3] Ping Y, Kai H, Qian Z. The influence of psychological status and disease uncertainty on patients with tubal infertility based on Neuman theory [J/OL]. China Journal of Health Psychology: 1-11[2020-07-08]. http://kns.cnki.net/kcms/ detail/11.5257.R.20200611.1346.014.html.

[4] Yu PW, Ling YL, Yu LY, et al. Reliability and validity of the Chinese version of the Benefit Discovery Scale in breast cancer patients $[\mathrm{J}]$. Chinese Journal of Clinical Psychology,2013,21(3):410-413.

[5] Chong HW. Measurement and assessment methods of life quality. Yunnan University Press.1999.

[6] Chun XH. Study on the current status and influencing factors of medical treatment in elderly patients with type- 2 diabetes [D]. Jinzhou Medical University,2015.

[7] Mao MZ. Construction of a self-disclosure based breast cancer patient benefit discovery intervention program [D]. Anhui Medical University,2019.

[8] Hua RL. Effect of Newman System Nursing Model on the Life Quality and Unhealthy Emotions of Patients with Interventional Therapy for Advanced Liver Cancer [J]. Anhui Medical and Pharmaceutical Journal, 2019, 23(9): 1834-1838. 